Pacific Journal of Mathematics 


\title{
AFFINE OPEN ORBITS, REDUCTIVE ISOTROPY GROUPS, AND DOMINANT GRADIENT MORPHISMS; A THEOREM OF MIKIO SATO
}

\author{
Frank SeRvedio
}

\begin{abstract}
An algebraic proof is given for a theorem of M. Sato. The theorem gives criteria for the open orbit in a prehomogeneous vector space under a reductive group to be an affine variety. The following conditions are equivalent:

1. $O(G)$ the open orbit is an affine variety.

2. $G_{X}$ the isotropy subgroup of $X$ in $O(G)$ is reductive.

3. There exists a semi-invariant form $P$ of degree $r \geqq 2$ such that $\operatorname{grad} P: V \rightarrow V^{*}$ is a dominant morphism of affine varieties.
\end{abstract}

In 1965, Mikio Sato stated a theorem giving characterizations of open affine orbits in real or complex vector spaces under the actions of reductive linear Lie groups. The statement has not appeared published in a European language, but appeared as a remark in Japanese in [8]. "Let $(G, V)$ be a prehomogeneous pair; assume that $G$ is a reductive real or complex algebraic group. The following conditions are equivalent:

(i) $H_{X}$, the isotropy subgroup of $X$ in the open dense orbit, is reductive.

(ii) $S$, the union of singular $G$-orbits in $V$, is a union of hypersurfaces $Z\left(P_{1}\right) \cup Z\left(P_{2}\right) \cup \cdots \cup Z\left(P_{m}\right)$.

(iii) There exists a semi-invariant form $P$ for $G$ such that the mapping $\operatorname{grad} P / P: V-Z(P) \rightarrow V^{*}$ is dominant."

By a prehomogeneous pair $(G, V)$ we mean an algebraic subgroup $G \subseteq G L(V)$ acting on $V$, a finite dimensional vector space over $\boldsymbol{R}$ or $C$ such that there is an open dense orbit $O(G)$ in $V$; see [9]. A proof of the theorem was not known. The result is striking in that the conditions are superficially quite different; also they are entirely algebraic whereas the theorem appears in the Sugaku article [8] where the techniques are analytic. The theorem is restated and provided with an algebraic proof. The author wishes to gratefully acknowledge the observations and assistance of Takuro Shintani.

Let $k$ be an algebraically closed field of characteristic $0 . k$ shall denote the multiplicative group $k-\{0\} . \quad V$ shall always denote a finite dimensional $k$-vector space and $V^{*}$ shall be its $k$-dual. $G \subset G L(V)$ shall denote a closed algebraic subgroup defined over $k$. The topologies used are always the Zariski topologies on the spaces. A 
prehomogeneous pair $(G, V)$ is defined as above with this modification. Let $k[V]$ denote the graded affine $k$-algebra of polynomial functions on $V$. If $P \in k[V]$, reserve the notations " $Z(P)$ " for the Zariski closed subset of $V$ consisting of zeroes of the function $P$ and " $U_{P}$ " for the Zariski open subset $U_{P}=V-Z(P)$. If $P \neq 0, U_{P}$ is known to be an affine algebraic variety defined over $k$, Zariski dense in $V$; see [7]. Let " $O(G)$ " denote the Zariski open orbit of $G$ in $V$ for a prehomogeneous pair $(G, V) . G$ acts as a group of automorphisms of $k[V]$ by $\lambda_{g} P(X)=P\left(g^{-1} X\right)$ for all $g \in G, P \in k[V]$ and $X \in V . \quad P$ is semi-invariant for $G$ if there exists a $\chi \in k[G]$ which is a unit in $k[G]$ such that for all $g \in G, \lambda_{g} P=\chi(g)^{-1} P . \quad \chi: G \rightarrow k^{*}$ is a rational character. Define the morphism $\operatorname{grad} P: V \rightarrow V^{*}$ of the canonical affine variety structures on $V$ and $V^{*}$ by setting $(\operatorname{grad} P)(X)$ to be the element of $V^{*}$ given by $(\operatorname{grad} P)(X)(Z)=\left(D_{Z} P\right)(X)$, for all $Z \in V$, where $D_{z}: k[V] \rightarrow k[V]$ is the $k$-derivation of degree -1 on the $k$ algebra $k[V] . \quad k[V]$ is canonically isomorphic to the symmetric algebra $S_{k}\left(V^{*}\right)$ and in either description $D_{z}$ is defined by requiring $D_{z}(Y)=Y(Z)$ for all $Y \in V^{*}$. If a basis $\mathscr{B}=\left\{X_{1}, \cdots, X_{n}\right\}$ is chosen in $V$ and a dual basis $\mathscr{B}^{*}=\left\{Y_{1}, \cdots, Y_{n}\right\}$ in $V^{*}$ such that $Y_{j}\left(X_{i}\right)=$ $\delta_{i j}$, then $k[V]$ is naturally isomorphic to the polynomial algebra $k\left[Y_{1}, \cdots, Y_{n}\right]$ and $(\operatorname{grad} P)(X)=\sum_{i=1}^{n} \partial P / \partial Y_{i}(X) Y_{i}$, or in coordinates $(\operatorname{grad} P)(X)=\left(\partial P / \partial Y_{1}(X), \cdots, \partial P / \partial Y_{n}(X)\right)$.

SATO'S THEOREM. Let $(G, V)$ be a prehomogeneous pair such that $G$ is a reductive algebraic group containing $k \cdot I_{V}$. The following are equivalent:

(1) $O(G)$ is an affine variety defined over $k$.

(1') $O(G)$ is equal to $U_{P}$, for $P$ a nonzero semi-invariant form of degree $r \geqq 2$ for $G$.

(2) For $X \in O(G), G_{X}=\{g \in G \mid g X=X\}$, the subgroup fixing $X$ in $G$, is a reductive closed subgroup of $G$.

(3) There exists a nonzero form $P$ of degree $r \geqq 2$ in $k[V]$ semi-invariant for $G$ such that $\operatorname{grad} P: V \rightarrow V^{*}$ is a dominant morphism.

$\left(3^{\prime}\right)$ There exists a nonzero form $P$ in $k[V]$ of degree $r \geqq 2$ semi-invariant for $G$ such that $\operatorname{grad} P / P: V \rightarrow V^{*}, X \mapsto 1 / P(X)(\operatorname{grad} P)$ $(X)$ is a dominant rational mapping.

Remarks AND ExAmples. (a) The condition that $\operatorname{grad} P: V \rightarrow V^{*}$ is a dominant morphism is equivalent to the condition that the forms $\partial P / \partial Y_{i} ; i=1, \cdots, n$ be algebraically independent over $k$.

(b) Lemma. For a form $P \in k[V], \operatorname{grad} P: V \rightarrow V^{*}$ is a 
dominant morphism of affine algebraic varieties if and only if $\operatorname{grad} P / P: V \rightarrow V^{*}$ is a dominant rational mapping.

Proof. The proof is straightforward in view of the fact that the dominance of the rational mapping is equivalent to the algebraic independence of the rational functions $\partial P / \partial Y_{i} / P ; i=1, \cdots, n$.

This lemma enables us to conclude immediately that (3) and $\left(3^{\prime}\right)$ are equivalent.

(c) The theorem as stated in the Sugaku article [8], contains a "non-fatal" error. Statement "(ii)" lacks the requirement that $m$, the number of hypersurfaces, be greater than 1 or if $m=1$, that the degree of the form $P_{1}$ be greater than 1 .

(d) Examples. (i ) If $G=G L(V)$ and $\operatorname{dim} V \geqq 2$, then all statements $(1), \cdots,\left(3^{\prime}\right)$ are false; if $\operatorname{dim} V=1$, then all statements are true with $G=k^{*}, G_{X}=1$, and $P=Y_{1}^{2}$.

(ii) Let $R=Y_{1}^{2}+Y_{1}^{2}+\cdots+Y_{n}^{2}$ be a quadratic form on $k^{n}$, $G=k \cdot I_{V} \cdot O(n)$ where $O(n)$ is the orthogonal group of $R$. Then all statements of the theorem are true. (1) and $\left(1^{\prime}\right)$ are applications of Witt's theorem; $G_{X} \cong O(n-1)$ a reductive group and $\operatorname{grad} R$ gives a linear isomorphism since $R$ is a nondegenerate quadratic form.

(iii) For $V=k^{4 \times 3}$ and $G=k \cdot I_{V} \cdot \mathrm{Sp}(4) \times O(3)$ there is a semiinvariant form $P$ for $G$ of degree 4 . With $X=\left(X_{1}, X_{2}, X_{3}\right)$ and $X_{i} \in k^{4}, P(X)=\left[X_{1}, X_{2}\right]^{2}+\left[X_{2}, X_{3}\right]^{2}+\left[X_{3}, X_{1}\right]^{2}$ where [, $]$ is the skew bilinear form on $k^{4}$ defining the symplectic group, Sp(4). In this case we have

(1) $O(G)$ is not affine.

( $\left.1^{\prime}\right) \quad O(G) \varsubsetneqq U_{P}$; in fact $X=\left[\begin{array}{lll}1 & 0 & 0 \\ 0 & 0 & 0 \\ 0 & 1 & 0 \\ 0 & 0 & 0\end{array}\right] \in U_{P}$ but $G X$, the $G$-orbit of $X$ has codimension 2 in $U_{P} . \quad O(G) \subset U_{P}-G X$. of dimension 2 .

(2) For $X=\left[\begin{array}{lll}1 & 0 & 0 \\ 0 & 1 & 0 \\ 0 & 0 & 1 \\ 0 & 0 & 0\end{array}\right] \in O(G), G_{X}$ is a unipotent algebraic group

(3) $\operatorname{grad} P$ is not a dominant morphism. The closure of the image of $\operatorname{grad} P$ has codimension 2 in $V^{*}$. See [8], page 141 .

Proof of Sato's theorem. (1) if and only if (1'). Only "(1) implies ( $\left.1^{\prime}\right)$ " needs justification. Since $O(G)$ is open in $V$ and is an affine variety, by the result in [5], $V-O(G)$ is an algebraic set of 
pure codimension 1 . Since $k[V]$ is a unique factorization domain, $V-O(G)=Z(P)$ by [7]. Thus $O(G)=U_{P}$ for some $P \neq 0$ in $k[V]$. Clearly $P$ must be $G$-semi-invariant, and $P$ must be a form since $k \cdot I_{V} \subset G$. The form $P$ must have degree $r \geqq 2$; for if $r=1$, we may assume $P=Y_{1}$ and then $U_{P}=\left\{X \in V \mid Y_{1}(X) \neq 0\right\} . \quad Z\left(Y_{1}\right)=$ $\left\{X \in V \mid Y_{1}(X)=0\right\}$ is a $G$-invariant subspace of codimension 1 . Since $G$ is reductive there exists a complementary $G$-invariant subspace, a line $Z\left(Y_{2}, \cdots, Y_{n}\right)$ on appropriate choice of basis $\mathscr{B}$. But $Z\left(Y_{2}, \cdots, Y_{n}\right) \cap U_{Y_{1}}$ is nonempty unless $\operatorname{dim} V=1$, where the theorem has been verified. However, $Z\left(Y_{2}, \cdots, Y_{n}\right) \cap U_{Y_{1}}$ being nonempty contradicts $O(G)=U_{P}$.

(2) implies (1). Since $G_{X}$ is a closed subgroup of $G$ acting on $G$ by right translation and since $G_{X}$ is reductive, Mumford's theorem enables us to conclude that the quotient variety $G / G_{X}$ is an affine variety; see [4]. However, the action of $G_{X}$ on the image of the orbit mapping Gor: $X G \rightarrow O(G)$ is isomorphic

$$
g \longmapsto g X
$$

to the action of $G_{X}$ on $G$ by right translation and thus is a quotient morphism in the sense of [1]. Hence, $G / G_{X} \cong O(G)$. Therefore, $O(G)$ is affine.

(1) implies (2). As above, $G / G_{X} \cong O(G)$. With $G$ reductive and $k$ of characteristic $O$, and $O(G)$ affine, Theorem 3.5 in [2] allows us to conclude that $G_{X}$ is reductive.

The equivalence of (3) and conditions (1) and (2) is seen more easily if the following lemmas are established. First, fix some notation. Let $A \in \operatorname{Hom}_{k}(V, W)$, " $A^{*}$ " shall always denote the transpose of $A$. Thus $A^{*} \in \operatorname{Hom}_{k}\left(W^{*}, V^{*}\right)$ is defined by the requirement that $\left(A^{*} Y\right)(X)=Y(A X)$ for all $X \in V$ and all $Y \in W^{*}$.

LeMma 1. There is a k-linear isomorphism $T: V \rightarrow V^{*}$ such that $T^{*}=T$ and an automorphism $i: G \rightarrow G$ of order 2 over $k$ such that for all $g \in G$, and for all $X \in V, T(g X)=\left(i(g)^{*}\right)^{-1} T(X)$.

Proof. There is a $k=C$ version of this in [9], Lemma 1.1 on page 135. One can justify the result for $k$ by proving Lemma 2 below and then using it to obtain the result for $G$, whose Lie algebra is $L$ by imitating the techniques used in [10].

LEMMA 2. Let $L$ be a reductive algebraic Lie subalgebra of $L G L(V)$, the Lie algebra of $G L(V)$. There is a k-linear isomorphism $T: V \rightarrow V^{*}$ such that $T=T^{*}$ and a Lie algebra automorphism $i^{\prime}$ of $L$ of order 2 such that for all $A \in L$, for all $X \in V$, 
$T(A X)=-i^{\prime}(A)^{*} T(X)$.

Sketch of proof of Lemma 2. $L \cong \tau \times L^{\prime}$ where $\tau$ is an algebraic torus and $L^{\prime}$ is the derived subalgebra of $L, k$-split semi-simple; see [3]. For $i^{\prime}$, send elements of $\tau$ to their negatives and specify $i^{\prime}$ on $L^{\prime}$ by sending each root to its negative and extend on a system of canonical generators of $L^{\prime}$ as described in [6]. $T$ is specified by sending each element of a basis of weight vectors of $L^{\prime}$ in $V$ to its correspondent in a dual basis of $V^{*}$. This suffices to verify Lemma 2.

LeMMA 3. If $P$ is a semi-invariant form in $k[V]$ for $G$, then for all $g \in G$, for all $X, U \in V$, grad $P(g X)(g U)=\chi(g) \operatorname{grad} P(X)(U)$. Equivalently, for all $g \in G$, for all $X \in V, \quad \chi(g) g^{*-1} \operatorname{grad}(P)=$ $\operatorname{grad} P(g X)$.

Proof. Let $t$ be transcendental over $k$. $\operatorname{grad} P(X)(U)$ is the coefficient of $t$ in the $k[t]$-polynomial $P(X+t U)$; see [11]. The identity $\chi(g) P(X+t U)=P(g(X+t U))=P(g X+t g U)$ establishes the lemma.

Let $G^{*}=\left\{g^{*} \mid g \in G\right\}$. From Lemma 1, it follows that $\left(G^{*}, V^{*}\right)$ is a prehomogeneous pair. Let $O\left(G^{*}\right)$ be the open orbit in $V^{*}$. Since $k$ is algebraically closed and $T^{*}=T$, there exists a choice of basis $\mathscr{B}=\left\{X_{1}, \cdots, X_{n}\right\}$ such that $T \mathscr{B}=\left\{T X_{1}, \cdots, T X_{n}\right\}$ is the dual basis to $\mathscr{B}$, namely $\left(T X_{i}\right)\left(X_{j}\right)=\delta_{i j}$ for $i, j=1, \cdots, n$. Such a basis $\mathscr{B}$ will becalled an orthogonal basis. Any change of basis by an orthogonal transformation results again in an orthogonal basis. As above, let $\mathscr{B}^{*}=\left\{Y_{1}, \cdots, Y_{n}\right\}$ denote the dual basis of $\mathscr{B}$.

Lemma 4. For $(G, V)$ prehomogeneous with $G$ reductive and $P$ a semi-invariant for $G$, there exists an orthogonal basis $\mathscr{B}$ for $V$ with $X_{1} \in O(G)$ and $c \neq 0$ such that $\operatorname{grad} P\left(X_{1}\right)=c Y_{1}$ if and only if $\operatorname{grad} P: V \rightarrow V^{*}$ is a dominant morphism.

Proof. For a basis $\mathscr{B}$ let the $n \times 1$ matrix of coordinates or basis coefficients for $X \in V$ be denoted by $X_{-}$, the $n \times n$ matrix of $A \in \operatorname{End}_{k}(V)$ be denoted by $A_{s}$ and the $1 \times n$ matrix of dual basis coefficients of $Y \in V^{*}$ be denoted by $Y_{\mathscr{B}}$. Note that $Y(A X)=$ $Y_{\mathscr{S}} A_{\mathscr{B}} X_{\mathscr{B}}$. For an orthogonal basis $\mathscr{B}, Y_{\mathscr{A}}^{\text {transpose }}=\left(T^{-1} Y\right)_{\mathscr{B}}$. The conditions of Lemmas 1 and 3 give $i(g) X=T^{-1} g^{-1 *} T X$ and

$$
\operatorname{grad} P(g X)_{\mathscr{G}}^{\text {transpose }}=\left(\chi(g) T^{-1} g^{-1 *} \operatorname{grad} P(X)\right)_{\mathscr{A}} \text {. }
$$

Hence if $\mathscr{B}$ is an orthogonal basis and $X_{1} \in O(G)$ and $\operatorname{grad} P\left(X_{1}\right)=$ $c Y_{1}=c T X_{1}$ with $c \neq 0$, then 


$$
\operatorname{grad} P\left(g X_{1}\right)_{\mathscr{G}}^{\text {transpose }}=c\left(i(g) X_{1}\right)_{\mathscr{A}}=c i(g)_{\mathscr{S}} X_{1 \mathscr{P}} .
$$

Since $i$ is an automorphism of $G$ and $X_{1} \in O(G)$, the first column of coordinate functions of $G$ in basis $\mathscr{B}$ are algebraically independent. Hence the coordinate funetions of $\operatorname{grad} P$ are algebraically independent.

Conversely, if $\operatorname{grad} P$ is dominant, then the rational mapping $\operatorname{grad} P / P: V \rightarrow V^{*}$ has the property that $\operatorname{grad} P / P(O(G))$ contains a Zariski open subset $U$ of $V^{*}$ such that $k \cdot U \subset U$. Hence by the proposition below $\operatorname{grad} P / P(O(G))$ contains a vector $Y_{1}$ which may be completed to an orthogonal basis. Let $X_{1}$ be such that

$$
\frac{1}{r} \frac{\operatorname{grad} P}{P}\left(X_{1}\right)=Y_{1} \text {. }
$$

Since $O(G) \subset U_{P}$,

$$
Y_{1}\left(X_{1}\right)=\frac{1}{r} \frac{\operatorname{grad} P}{P}\left(X_{1}\right)\left(X_{1}\right)=1 .
$$

Now complete $\left\{X_{1}\right\}$ to an orthogonal basis for $V$.

Proposition. Let $U$ be a Zariski open subset of $V$ such that $k^{\cdot} U \subset U$, and let $R$ be a nondegenerate quadratic form on $V$. Then $U$ contains an orthogonal basis with respect to $R$.

Proof. $U \cap U_{R}$ is open and nonempty. Therefore there is an $X_{1} \in U \cap U_{R}$ such that $R\left(X_{1}\right)=1$. Let $Y_{1}=R\left(X_{1}, \quad\right)$ be the linear (polynomial) function on $V$ given by the symmetric bilinear form associated to $R . Z\left(Y_{1}\right)$ is the closed subset of $V$ with underlying point set equal to the vector space $Y_{1}^{\perp} . \quad R_{1}\left(=R\right.$ restricted to $\left.Y_{1}\right)$ is a nondegenerate quadratic form. Consider $U \cap U_{R} \cap Z\left(Y_{1}\right)$. If the latter is nonempty choose $X_{2}$ as above in the choice of $X_{1}$ for this vector space $Y_{1}^{\perp}$. If $U \cap U_{R} \cap Z\left(Y_{1}\right)$ is empty, then $Z\left(Y_{1}\right) \subset Z(R) \cup S$, where $S=V-U . \quad Z\left(Y_{1}\right)$ is an irreducible closed set. Hence $Z\left(Y_{1}\right) \subset S=Z\left(R_{1}, \cdots, R_{m}\right)$, where $R_{i}, i=1, \cdots, m$ are forms in $k[V]$. Equivalently the following inclusion of ideals holds;

$$
\left(Y_{1}\right) \supset\left(R_{1}, \cdots, R_{m}\right) \text {. }
$$

It is clear now that an $X_{1}^{\prime}$ could be chosen, as was $X_{1}$ for which $\left(Y_{1}^{\prime}\right) \not \supset\left(R_{1}, \cdots, R_{m}\right)$ so that $U \cap U_{R} \cap Z\left(Y_{1}^{\prime}\right)$ is not empty. Proceed inductively until an orthogonal basis is chosen in $V$.

In characteristic 0 , it is well known that if a closed algebraic subgroup of $G L(V)$ has a reductive Lie algebra, then that subgroup is reductive; see [4], Proposition 3.31 and [3]. 
(3) implies (2). For this part of the proof we use the lie algebras of $G L(V), G$ and $G_{X}$ which we denote by $L G L(V), L$ and $L_{X}$ respectively. These are algebraic Lie algebras over $k$. We show $G_{X}$ reductive by showing $L_{X}$ reductive. $L X=\{A X \mid A \in L\}$ is canonically isomorphic to the tangent space of the orbit $G X$ at $X$. Hence $L_{X}=\{A \in L \mid A X=0\}$. For $X \in O(G), L_{X}$ has codimension $n$ in $L$ since the dimension of the orbit $O(G)=G X$ is $n$. We use the following criterion of reductivity for algebraic Lie algebras.

LEMMA 5. Let $L \subset L G L(V)$ be an algebraic Lie subalgebra. $L$ is reductive if and only if the trace form restricted to $L \times L$ is nondegenerate.

\section{Proof. See [3].}

The trace form is nondegenerate when restricted to $L$. We need show that the trace form restricted to $L_{X}$ is nondegenerate for $X \in O(G)$. We show that $L_{X}$ can be defined under the trace form as the subspace orthogonal and complementary to a subspace of $L$ of codimension $n$. As above, choose an orthogonal basis where $X_{1} \in O(G)$ and $\operatorname{grad} P\left(X_{1}\right)=c Y_{1}, c \neq 0$. Since $\operatorname{grad} P(g X)=\chi(g) g^{*-1} \operatorname{grad} P(X)$, we see that $L_{X}=L_{\operatorname{grad} P(X)}$ where $L_{\operatorname{grad} P(X)}=\left\{A \in L \mid A^{*} \operatorname{grad} P(X)=0\right.$ in $V$ \}. $\operatorname{grad} P$ is dominant implies that $\operatorname{grad} P\left(X_{1}\right)$ lies in the open orbit $O\left(G^{*}\right)$ in $V^{*}$ and hence $L_{\mathrm{grad} P\left(X_{1}\right)}$ is also of codimension $n$ in $L$. Hence $L_{X_{1}}=L_{\operatorname{grad} P\left(X_{1}\right)}$. With the basis chosen as above, $A X_{1}=0$ if and only if $A_{i 1}=0$ for $i=1, \cdots, n$ if and only if Trace $A E_{1 j}=0$ for $j=1, \cdots, n$ where $E_{1 j}$ is the $n \times n$ matrix with first row $(0, \cdots, 0,1,0, \cdots, 0)$ with 1 in the $j$ th place and other rows zero if and only if Trace $A E_{1 j}^{\prime}=0$ where $E_{1 j}^{\prime} \equiv E_{1 j}$ modulo the annihilator of $L$ under the trace form and $E_{1 j}^{\prime} \in L$. Let $M_{X_{1}}$ be the subspace spanned by $E_{1 j}^{\prime}$ in $L$. The criterion $L_{X_{1}}=L_{Y_{1}}$ implies immediately that $L_{X_{1}} \cap M_{X_{1}}=0$. Hence $L_{X_{1}}$ is reductive.

( $\left.1^{\prime}\right)$ implies (3). We assume that $O(G)=U_{P}$. Recall that the dual pair $\left(G^{*}, V^{*}\right)$ is a prehomogeneous vector space with a corresponding form $Q \in k\left[V^{*}\right]$ of degree $r$; Lemma 1 gives this. $\operatorname{grad} P$ sends $G$ orbits to $G^{*}$ orbits; i.e., grad $P(G X)=G^{*} \operatorname{grad} P(X)$ for all $X \in V$. Lemma 3 implies this easily. Let $R$ be the quadratic form associated to the $k$-vector space mapping $T: V \rightarrow V^{*}$ of Lemma 1 , so that $R(X)=T(X)(X)$. We may choose an $X_{1} \in O(G) \cap U_{R}$ and assume that $P\left(X_{1}\right)=1$ and that $X_{1}$ is a member of an orthogonal basis $\mathscr{B}$. Then $P=Y_{1}^{r}+Y_{1}^{r-1} P_{1}+\cdots+Y_{1} P_{r-1}+P_{r}$ with $P_{i} \in$ $k\left[Y_{2}, \cdots, Y_{n}\right]$ of degree $i$. We compute easily that $\operatorname{grad} P\left(X_{1}\right)=$ $r Y_{1}+P_{1}$. Since $\mathscr{B}$ is an orthogonal basis, $Q=X_{1}^{r}+X_{1}^{r-1} Q_{1}+\cdots+$ $X_{1} Q_{r-1}+Q_{r}$ where $Q_{i} \in k\left[X_{2}, \cdots, X_{n}\right]$ is of degree $i$ and is the cor- 
respondent of $P_{i}$. Thus $Q_{i}$ is $P_{i}$ with $Y$ replaced by $X$. We establish that $Q\left(\operatorname{grad} P\left(X_{1}\right)\right) \neq 0$. For any $g \in G$,

$$
Q\left(\operatorname{grad} P\left(g X_{1}\right)\right)=Q\left(\chi(g) g^{-1} * \operatorname{grad} P\left(X_{1}\right)\right)=\chi(g)^{r} Q\left(g^{-1} *\left(r Y_{1}+P_{1}\right)\right) .
$$

It suffices to compute $Q\left(g *\left(r Y_{1}+P_{1}\right)\right)$.

$$
\begin{aligned}
Q(g *( & \left.\left.r Y_{1}+P_{1}\right)\right)=X_{1}^{r}\left(g *\left(r Y_{1}+P_{1}\right)\right) \\
& +X_{1}^{r-1}\left(g *\left(r Y_{1}+P_{1}\right)\right) Q_{1}\left(g *\left(r Y_{1}+P_{1}\right)\right)+\cdots+Q_{r}\left(g *\left(r Y_{1}+P_{1}\right)\right) \\
= & \left(g X_{1}\right)^{r}\left(r Y_{1}+P_{1}\right)+\left(g X_{1}\right)^{r-1}\left(r Y_{1}+P_{1}\right) g Q_{1}\left(r Y_{1}+P_{1}\right) \\
& +\cdots+g Q_{r}\left(r Y_{1}+P_{1}\right) \\
= & \left(\sum_{i=1}^{n} g_{i 1} X_{i}\right)^{r}\left(r Y_{1}+P_{1}\right)+\left(\sum_{i=1}^{n} g_{i 1} X_{i}\right)^{r-1}\left(r Y_{1}+P_{1}\right) g Q_{1}\left(r Y_{1}+P_{1}\right) \\
& +\cdots+g Q_{r}\left(r Y_{1}+P_{1}\right) \\
= & \left(r g_{11}+\sum_{i=2}^{n} g_{i 1} X_{i}\left(P_{1}\right)\right)^{r}+\left(r g_{11}+\sum_{i=2}^{n} g_{i 1} X_{i}\left(P_{1}\right)\right)^{r-1} g Q_{1}\left(r Y_{1}+P_{1}\right) \\
& +\cdots+g Q_{r}\left(r Y_{1}+P_{1}\right) .
\end{aligned}
$$

The latter is a nonzero polynomial expression of the type

$$
r^{r} g_{11}^{r}+g_{11}^{r-1} S_{r-1}(g)+\cdots+g_{11} S_{1}(g)+S_{0}(g)
$$

with $S_{i}(g)$ polynomial expressions in the coordinate functions $g_{1 m}$ with $(1, m) \neq(1,1)$. This polynomial cannot be the zero polynomial, since otherwise $g_{11}$ is algebraically dependent on the $g_{1 m}$ with $(1, m) \neq(1,1)$ and this contradicts that the point $X_{1} \in O(G)$. This completes the proof of the theorem.

A description of all prehomogeneous pairs $(G, V)$ over $k$ with $G$ acting irreducibly on $V$ is being sought. The examples such as (iii) with $\operatorname{Sp}(2 n), n \geqq 2$, are the only ones known where there exists a semi-invariant $P$ and the condition $O(G) \varsubsetneqq U_{P}$ maintains. We have shown that grad $P(O(G))$ is contained in a proper $G$-invariant closed subvariety of $U_{Q}$ in $V^{*}$. In general grad $P$ restricted to $Z(P)$ fails to have the property of being a dominant mapping to $Z(Q)$ even when the conditions of the theorem hold; an example is $G \cong k^{*}$. $S L(n) \times S L(n)$ acting on $k^{n \times n}$ with $\left(c, g_{1}, g_{2}\right) X=c g_{1} X g_{2}^{-1}$ and $P=$ determinant.

\section{REFERENCES}

1. Armand Borel, Linear Algebraic Groups, 1969, W. A. Benjamin, (6.1) and (6.8).

2. Armand Borel and Harish Chandra, Arithmetic subgroups of algebraic groups, Ann. of Math., 75 no. 3, (1962), Theorem 3.5.

3. Bourbaki, Elements of Mathematics, Lie Groups and Lie Algebras, part I, AddisonWesley Publishing Company, 1975. Ch. I, §6.4.

4. John Fogarty, Invariant Theory, W. A. Benjamin, 1969, p. 159. 
5. Jacob Eli Goodman, Affine open subsets of algebraic varieties, Ann. of Math., 89 (1969), 162.

6. Nathan Jacobson, Lie Algebras, Interscience Publishers, 1962, Ch. IV, §3 and Theorem 3, 127.

7. David Mumford, Introduction to Algebraic Geometry, Harvard University Lecture Notes, Propn. 4, p. 87.

8. Mikio Sato, The theory of prehomogeneous vector spaces, Sugaku no Ayumi 15-1 (1970), 85-157, notes by T. Shintani.

9. Takuro Shintani, On Dirichlet series whose coefficients are class numbers of integral binary cubic forms, J. Math. Soc. Japan, 24, no. 1, 132-188.

10. R. Steinberg, Lectures on Chevalley Groups, Yale University Lecture Notes, 1968, Theorem 29.

11. Hermann Weyl, The Classical Groups, Princeton University Press, 1946, p. 5.

Received November 4, 1976 and in revised form March 23, 1977.

MCMASTER UNIVERSITY

Hamilton, Ontario, Canada 



\title{
PACIFIC JOURNAL OF MATHEMATICS
}

\section{EDITORS}

\author{
RICHARD ARENS (Managing Editor) \\ University of California \\ Los Angeles, CA 90024 \\ Charles W. Curtis \\ University of Oregon \\ Eugene, OR 97403 \\ C. C. MOORE \\ University of California \\ Berkeley, CA 94720
}

\section{J. DUGUNDJI}

Department of Mathematics

University of Southern California

Los Angeles, CA 90007

R. FINN and J. MILGRAM

Stanford University

Stanford, CA 94305

\section{ASSOCIATE EDITORS}
E. F. BECKENBACH
B. H. NeumanN
F. WOLF
K. YoSHIDA

\section{SUPPORTING INSTITUTIONS}

UNIVERSITY OF BRITISH COLUMBIA

CALIFORNIA INSTITUTE OF TECHNOLOGY

UNIVERSITY OF CALIFORNIA

MONTANA STATE UNIVERSITY

UNIVERSITY OF NEVADA, RENO

NEW MEXICO STATE UNIVERSITY

OREGON STATE UNIVERSITY

UNIVERSITY OF OREGON

OSAKA UNIVERSITY

\author{
UNIVERSITY OF SOUTHERN CALIFORNIA \\ STANFORD UNIVERSITY \\ UNIVERSITY OF HAWAII \\ UNIVERSITY OF TOKYO \\ UNIVERSITY OF UTAH \\ WASHINGTON STATE UNIVERSITY \\ UNIVERSITY OF WASHINGTON \\ $\stackrel{*}{*} \stackrel{*}{*} \stackrel{*}{*}$ AMERICAN MATHEMATICAL SOCIETY
}

The Supporting Institutions listed above contribute to the cost of publication of this Journal, but they are not owners or publishers and have no responsibility for its content or policies.

Mathematical papers intended for publication in the Pacific Jaurnal of Mathematics should be in typed form or offset-reproduced, (not dittoed), double spaced with large margins. Please do not use built up fractions in the text of your manuscript. You may however, use them in the displayed equations. Underline Greek letters in red, German in green, and script in blue. The first paragraph or two must be capable of being used separately as a synopsis of the entire paper. Items of the bibliography should not be cited there unless absolutely necessary, in which case they must be identified by author and Journal, rather than by item number. Manuscripts, in triplicate, may be sent to any one of the editors. Please classify according to the scheme of Math. Reviews, Index to Vol. 39. All other communications should be addressed to the managing editor, or Elaine Barth, University of California, Los Angeles, California, 90024.

The Pacific Journal of Mathematics expects the author's institution to pay page charges, and reserves the right to delay publication for nonpayment of charges in case of financial emergency.

100 reprints are provided free for each article, only if page charges have been substantially paid. Additional copies may be obtained at cost in multiples of 50 .

The Pacific Journal of Mathematics is issued monthly as of January 1966. Regular subscription rate: $\$ 7200$ a year (6 Vols., 12 issues). Special rate: $\$ 36.00$ a year to individual members of supporting institutions.

Subscriptions, orders for back numbers, and changes of address should be sent to Pacific Journal of Mathematics, 103 Highland Boulevard, Berkeley, California, 94708.

PUBLISHED BY PACIFIC JOURNAL OF MATHEMATICS, A NON-PROFIT CORPORATION

Printed at Kokusai Bunken Insatsusha (International Academic Printing Co., Ltd.). 8-8, 3-chome, Takadanobaba, Shinjuku-ku, Tokyo 160, Japan.

Copyright (C) 1975 by Pacific Journal of Mathematics Manufactured and first issued in Japan 


\section{Pacific Journal of Mathematics}

Vol. 72, No. 2

February, 1977

George E. Andrews, Plane partitions. II. The equivalence of the

Bender-Knuth and MacMahon conjectures ................. 283

Lee Wilson Badger, An Ehrenfeucht game for the multivariable quantifiers

of Malitz and some applications ......................... 293

Wayne C. Bell, A decomposition of additive set functions ............ 305

Bruce Blackadar, Infinite tensor products of $C^{*}$-algebras ............. 313

Arne Brøndsted, The inner aperture of a convex set .............. 335

N. Burgoyne, Finite groups with Chevalley-type components........... 341

Richard Dowell Byrd, Justin Thomas Lloyd and Roberto A. Mena, On the retractability of some one-relator groups .....................

Paul Robert Chernoff, Schrödinger and Dirac operators with singular potentials and hyperbolic equations .................... 361

John J. F. Fournier, Sharpness in Young's inequality for convolution ....... 383

Stanley Phillip Franklin and Barbara V. Smith Thomas, On the metrizability

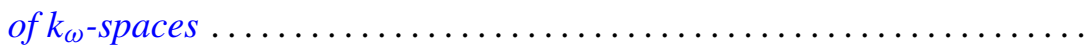

David Andrew Gay, Andrew McDaniel and William Yslas Vélez, Partially normal radical extensions of the rationals .................... 403

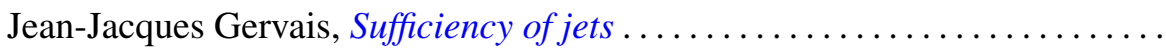

Kenneth R. Goodearl, Completions of regular rings. II . .

Sarah J. Gottlieb, Algebraic automorphisms of algebraic groups with stable maximal tori

Donald Gordon James, Invariant submodules of unimodular Hermitian forms.....

J. Kyle, $W_{\delta}(T)$ is convex.

Ernest A. Michael and Mary Ellen Rudin, A note on Eberlein compacts ...

Ernest A. Michael and Mary Ellen Rudin, Another note on Eberlein compacts ....

Thomas Bourque Muenzenberger and Raymond Earl Smithson, Fixed point theorems for acyclic and dendritic spaces.

Budh Singh Nashier and A. R. Rajwade, Determination of a unique solution of the quadratic partition for primes $p \equiv 1(\bmod 7)$.

Frederick J. Scott, New partial asymptotic stability results for nonlinear ordinary differential equations ....................

Frank Servedio, Affine open orbits, reductive isotropy groups, and dominant gradient morphisms; a theorem of Mikio Sato..........

D. Suryanarayana, On the distribution of some generalized square-full integers.................................. 